\title{
MamáFeliz*: una aproximación teórica de las TIC aplicadas a la prevención de la depresión posparto
}

\author{
CRISTIAN CAStELLANO BADENAS \\ al107103@alumail.uji.es \\ ELENA FERMOSELLE Esclapez \\ al076962@alumail.uji.es \\ JORGE JAVIER OSMA LÓPEZ \\ osma@psb.uji.es \\ Elena CRespo Delgado \\ screspo@psb.uji.es \\ JUAN RAMÓN BARREDA \\ barrada@unizar.es
}

\section{Resumen}

La evaluación, diagnóstico y tratamiento psicológico a través de las tecnologías de la información y la comunicación (TIC) es una realidad cada vez más frecuente en la Psicología Clínica y de la Salud. Las posibilidades ilimitadas que ofrece Internet han hecho posible que ya existan estudios controlados sobre intervenciones basadas en aplicaciones online para la prevención y tratamiento de trastornos mentales y para la promoción de conductas de salud.

Las alteraciones emocionales en la mujer asociadas al periodo perinatal y al puerperio -especialmente la Depresión Posparto (DPP), depresión prenatal (DP) y tristeza posparto (TPP) - suponen un gran problema de salud, dada su prevalencia en población general, su riesgo de cronicidad y el coste que de ello se deriva. Los datos de prevalencia de la DPP en España ofrecen porcentajes situados entre el 6-25\% según algunos estudios. A pesar de estos datos, las alteraciones emocionales perinatales siguen pasando desapercibidas, infradiagnosticadas y, por tanto, no tratadas.

*MamáFeliz es un proyecto subvencionado por la Conselleria de Sanitat de la Generalitat Valenciana, el Gobierno de Aragón, la Fundación Universitaria Antonio Gargallo (Universidad de Zaragoza. Campus de Teruel) y CREOS, centro de psicoterapia y formación (Castellón).

Agradecimiento: El equipo investigador agradece la colaboración por parte de los diferentes profesionales sanitarios de los centros colaboradores de MamáFeliz: Hospital La Plana Vila-real, Hospital NISA Rey Don Jaime de Castellón, Centro de Salud Teruel Ensanche, Centro de Salud Picarral (Zaragoza), Centro de Salud Pirineos (Huesca), Grupo Aragonés de investigación en Atención Primaria, Grupo Aragonés de investigación de salud mental en Atención Primaria. 
La inconsistencia de los resultados de diferentes estudios sobre los factores de riesgo implicados requiere homogeneizar su medición. Asimismo, los recursos asistenciales en la salud pública son limitados y se hace necesario mejorar la calidad de los mismos y su eficacia reduciendo los costes. Con este objetivo, hemos diseñado Mamáfeliz (MMF), una herramienta informática que, a través de Internet, realiza de manera rápida y sencilla una evaluación exhaustiva a nivel psicosocial, obstétrico y psicopatológico con el fin de identificar aquellas mujeres que presentan uno o más factores de riesgo para el desarrollo de DPP.

Palabras clave: depresión posparto, factores de riesgo, TIC, embarazo, prevención, promoción de la salud.

\section{Abstract}

The assessment, diagnosis and psychological treatment throught Information and communications technology (ICTS) are a reality more frequent in Clinical and Health Psychology. Unlimited possibilities offered by the Internet have made the existence of controlled studies about interventions based on on-line applications for the prevention and treatment of mental disorders and for the promotion of healthy conducts possible.

Emotional variations in women which are associated with the perinatal period and peuperium, specially Postpartum Depression (PPD), Prenatal Depression (PD) and Baby Blues (BB), mean a big health problem, given its prevalence among the general population, its risk of chronicity and the derived cost. The data of the PND prevalence in Spain shows percentages between 6-25\% according to some studies.

The obtained results from different studies about implicated risk factors show an inconsistency which is required to level down the risk factor its measurement. Just like that, assistance means in the public health are limited and that make necessary improve their quality and efficiency by reducing the costs. So, in order to achieve this goal, we have designed Mamáfeliz (MMF), an informatic tool that through the Internet carries out an exhaustive evaluation in a fast and simple way at a psychosocial, obstetrical and psychopathologic level in order to identify those women who present one or more risk factors for the PPD development.

Keywords: Postpartum depresión, risk factors, ICTS, pregnancy, prevention, health promotion.

\section{Introducción}

Existe unanimidad en la comunidad científica acerca de la necesidad de mejorar y establecer nuevas herramientas en los protocolos de evaluación, diagnostico e intervención, en todas las áreas de la atención sanitaria. En este sentido, las TIC pueden proporcionarnos numerosas posibilidades y ventajas, hasta ahora desconocidas e infrautilizadas, para alcanzar los niveles de excelencia necesarios en cuanto a efectividad, eficacia y eficiencia de las intervenciones aplicadas. Este objetivo de innovación, acerca de la mejora de la asistencia proporcionada desde algunas áreas de salud, junto con las líneas de trabajo promovidas por el e- 
Health Action Plan (e-HAP) de la UE 2012-2020, coinciden en resaltar la importancia de la mejora en la calidad y la gestión sanitaria a partir del estudio comparativo de los sistemas sanitarios y de las intervenciones y servicios ofrecidos por los mismos (Jané-Llopis, 2004). En este sentido, la psicología ha sido una de las primeras disciplinas de la rama sanitaria que ha realizado grandes esfuerzos para establecer y diseñar protocolos de evaluación y tratamiento de manera computerizada. Hasta el momento, los resultados obtenidos por este tipo de herramientas autoaplicadas en psicoterapia, han resultado ser tan eficaces como los obtenidos por los tratamientos aplicados a través del método tradicional (Andersson, 2009; Andersson y Carlbring, 2006; Ritterband et al., 2003). Además, para ampliar las oportunidades y ventajas potenciales que pueden aportar las TIC en los servicios sanitarios y las intervenciones implementadas en los mismos, resultaría de gran utilidad dotar a este tipo de herramientas las máximas funciones posibles. Centrándonos no solo en su función como herramienta de prevención o diagnóstico, sino dotándolas de otras utilidades para los servicios de salud como: 1) servicios no ofrecidos en los sistemas de salud; 2) proporcionar un nexo de comunicación más rápido y directo entre los profesionales, los usuarios y las instituciones; 3) tomar en consideración la información acerca de la aceptación y la utilidad percibida por parte de los usuarios, profesionales e instituciones; 4) llegar al mayor número posible de potenciales beneficiarios; entre otras muchas ventajas.

\section{Promoción de la salud mental y prevención de los trastornos psicológicos}

La promoción de la salud y la prevención son actividades necesariamente relacionadas que tienen un objetivo común: mejorar la salud mental (Jané-Llopis, 2004). La carta de Ottawa de Promoción de la Salud (OMS, 1986), sostiene que la prevención de los trastornos mentales se caracteriza por su enfoque en la reducción de factores de riesgo y la promoción de factores de protección relacionados con un trastorno mental o problema de conducta, con el objetivo final de reducir su prevalencia e incidencia (Jané-Llopis, 2004). Existe suficiente evidencia de que los programas de prevención y promoción funcionan y producen beneficios importantes en la salud de los individuos y en general en toda la sociedad (Hosman y Jané-Llopis, 1999; Mrazek y Hall, 1997).

Desde diferentes instituciones internacionales responsables en materia sanitaria se han promovido diferentes iniciativas como el Libro Verde de la Salud Mental (UE, 2005) o el Pacto Europeo para la Salud Mental y el Bienestar (UE, 2008); desde todas ellas se ha puesto de manifiesto la necesidad de desarrollar acciones eficaces para mejorar la Salud Mental de la población y han reconocido, como primera línea prioritaria de acción, la prevención de la depresión y del suicidio. Con este objetivo, instan al diseño y desarrollo de medidas para reducir los factores de riesgo de la depresión y el suicidio, incluyendo el abordaje de la Depresión Posparto (DPP) de las madres como uno de los temas concretos a tratar.

En España, desde 2007, la Estrategia Nacional de Salud Sexual y Reproductiva (ENSSR), ha desarrollo e implantado en el Sistema Nacional de Salud (SNS) la Estrategia de Atención al Parto Normal (EAPN) que en su objetivo específico n. ${ }^{\circ} 4$ apuesta por "promover la salud y el bienestar de las mujeres puérperas, facilitando asesoramiento y cuidados, explorando sus necesidades, cambios emocionales y psicológicos». Dentro de la propia ENSSR (2010), se hace referencia a la necesidad de atender, de manera paralela, al proceso fisiológico del embarazo y al proceso psicológico que lo acompaña e inciden en la necesidad de apoyar estudios sobre los aspectos en los que existe un vacío o menor conocimiento disponible, tales como: 1) la promoción del bienestar psicológico y emocional de las gestantes durante el embarazo; 
2) el establecimiento de rutinas de exploración de bienestar emocional de la puérpera, y 3) la prevención e identificación precoz de la psicopatología del posparto.

\section{Salud mental reproductiva en la mujer: Alteraciones emocionales en el periodo prenatal y perinatal}

Una de las líneas de interés por parte de los investigadores se ha focalizado en el bienestar psicológico de la mujer, realizando esfuerzos por el estudio de las alteraciones emocionales que se producen en el periodo prenatal y postnatal. Uno de los motivos más importantes es su alta prevalencia (Gaynes, Gavin y Meltzer-Brody et al., 2005) dado que, los trastornos del estado de ánimo son los más frecuentes a las seis semanas del parto, con una prevalencia del 10,1\%, seguidos por los trastornos adaptativos con un 4,4\%, y finalmente por los trastornos de ansiedad con un 4,2 \% (García-Esteve, Ascaso, García y Tamayo, 2003). Centrándonos únicamente en la DPP, desafortunadamente pasa desapercibida y por tanto no tratada en muchas ocasiones. Aproximadamente la mitad de las mujeres con DPP no reciben ningún tipo de evaluación psicológica o tratamiento (US Departament Health and Human Services, 2000), y solo el $23 \%$ de las mujeres diagnosticadas con depresión menor o mayor, recibe algún tipo de cuidado psicológico durante el embarazo (Kelly, Zatzick y Anders, 2001). La prevalencia e incidencia de las alteraciones emocionales de la mujer en el puerperio, las consecuencias en ellas mismas y sus descendientes así como familiares más directos, constituyen un problema general de salud (Pop, Essed, De Geus, Van Son y Komproe, 1993) e implican un incremento significativo en los costes sanitarios (Dosset, 2008; Oates, et al., 2004; Tortajada et al., 2009).

Todas estas razones subrayan la necesidad de realizar mejoras en nuestros actuales servicios públicos y privados de salud orientadas a: mejorar las estrategias de detección de mujeres con factores de riesgo para desarrollar una depresión, desarrollar intervenciones preventivas eficaces durante el periodo prenatal y postnatal y mejorar los recursos y herramientas orientados a la detección precoz de mujeres que presentan sintomatología depresiva durante o después del parto para su rápida derivación, evitando así la cronificación de los síntomas y sus consecuencias.

\section{Nuevas tecnologías en la prevención de trastornos mentales en la mujer: la DPP}

La evaluación e intervención psicológica a través de internet o también conocida como ciberterapia (e-terapia, terapia on line, terapia por ordenador), se está transformando en un complemento y una alternativa a la atención tradicional, y está comenzando a demostrar su eficacia (Soto-Pérez, Franco, Monardes y Jiménez, 2010). Para Andersson (2009), estamos asistiendo al nacimiento de una nueva forma de intervención: la intervención a través de internet. Ya existen estudios controlados sobre intervenciones basadas en aplicaciones web y/o terapia por vídeoconferencia para la prevención y tratamiento de trastornos mentales y para la promoción de conductas de salud (Mitchell, Stanimirovic, Klein y Vella-Brodrick, 2009), algunos ejemplos son: Walters, Miller y Chiauzzi (2005) para el abuso de alcohol; Proudfoot et al., (2003) para ansiedad; Proudfoot et al., (2007) para el trastorno bipolar; Anderson et al., (2005) para la depresión; Anderson et al., (2006) para la fobia social; Pier et al., (2008) para el trastorno de pánico; Spittaels y De Bourdeaudhuij (2006) para promover la actividad física, y Walters, Wright y Shegog (2006) para dejar de fumar, entre otros muchos.

Como ya hemos dicho las intervenciones psicológicas online han demostrado su eficacia como medio para el tratamiento de diferentes trastornos mentales. En la tabla 1, hemos reco- 
gido las principales ventajas de los programas e-Health junto con sus posibles limitaciones (Bennet, Casebeer, Zheng y Kristofco, 2004, 2006; Choque, 2010).

Tabla 1

Principales ventajas y limitaciones de los programas e-Health (Bennet et al. 2004, 2006; Choque, 2010)

\begin{tabular}{|c|c|}
\hline VENTAJAS & LIMITACIONES \\
\hline $\begin{array}{l}\text { - Se ha demostrado su idoneidad como for- } \\
\text { mato de evaluación psicológica. } \\
\text { - Permiten llegar a un mayor número de } \\
\text { beneficiarios, de manera más rápida y có- } \\
\text { moda. } \\
\text { - Proporcionan mayor privacidad y autono- } \\
\text { mía al usuario/a objetivo. } \\
\text { - Generan nuevas fórmulas de comunicación } \\
\text { y de intervención. } \\
\text { - Proporcionan productos dinámicos, siem- } \\
\text { pre dispuestos a incorporar nuevas funcio- } \\
\text { nalidades y mejoras. } \\
\text { - Su uso constituye un avance irrenunciable } \\
\text { acorde al momento tecnológico actual } \\
\text { - Su diseño reinterpreta y mejora las limita- } \\
\text { ciones de los programas en formato tradi- } \\
\text { cional. } \\
\text { - Permiten implementar actuaciones aun } \\
\text { disponiendo de escasos recursos económi- } \\
\text { cos. }\end{array}$ & $\begin{array}{l}\text { - Falta de estudios concluyentes que determinen } \\
\text { de manera firme su idoneidad como formato } \\
\text { empíricamente validado. } \\
\text { - Falta de coordinación de los servicios disponi- } \\
\text { bles de atención a la mujer embarazada. } \\
\text { - Falta de consistencia en las medidas empleadas, } \\
\text { el diseño de investigación y la temporalidad de } \\
\text { las mediciones que permitan replicar los resulta- } \\
\text { dos obtenidos o comparar los hallazgos encon- } \\
\text { trado a lo largo de los diferentes estudios reali- } \\
\text { zados a nivel mundial } \\
\text { - Ninguno de los programas en formato e-Health } \\
\text { reúne la evaluación de todos los FR recogidos a } \\
\text { lo largo de la literatura científica. } \\
\text { - Focalizan su implementación por parte de grupos } \\
\text { de población específicos (entornos rurales, nivel } \\
\text { socioeconómico bajo, riesgo de exclusión social, } \\
\text { etc.). }\end{array}$ \\
\hline
\end{tabular}

Dado que la depresión es uno de los trastornos mentales con mayor prevalencia en el mundo, en los últimos años se han diseñado y desarrollado numerosas aplicaciones web y herramientas informáticas de autoayuda para promover el bienestar y contrarrestar toda la sintomatología característica de los trastornos del estado de ánimo. Algunas de estas herramientas dirigidas a la evaluación y el tratamiento de la depresión han mostrado eficacia y efectividad en su aplicación en diferentes poblaciones (adultos, estudiantes universitarios y adolescentes), como muestran los resultados obtenidos por Proudfoot et al. (2003) con su ampliamente conocido cyber-programa «Beating the Blues». Este tratamiento on line ha mostrado buenos resultados y se ha posicionado como una iniciativa referente en este tipo de intervenciones psicológicas online, puesto que ha sido incluido en la guía del National Institute for Health and Clinical Excellence (NICE). Otras herramientas informáticas sobre el tratamiento de la depresión son los programas australianos «BluePages», desarrollados por el grupo de Hampton (2006), y el programa «MoodGYM», trabajo realizado en Suecia con las aportaciones de Andersson et al. (2005).

Si nos centramos específicamente en las intervenciones realizadas para evaluar o tratar a mujeres con DPP a través de las TIC observaremos la escasa investigación dedicada a pesar de tratarse de un problema clínico significativo y muy prevalente (Le, Perry y Sheng, 2009). Le et al. (2009) examinaron en su trabajo la viabilidad de utilizar internet para rastrear síntomas de DPP mediante la cumplimentación de los instrumentos PDSS (Postpartum Depression Screening Scale; Beck y cols., 2001) y EPDS (Edinburg Postpartum Depression Scale; Cox, Holden y Sagovsky, 1987) a través de internet. Los resultados obtenidos por este grupo de la George 
Washington University, a pesar de una serie de limitaciones, mostraron la idoneidad de internet como plataforma de evaluación para este tipo de sintomatología. Las limitaciones vienen dadas, en este proyecto, por la no inclusión de otros aspectos a evaluar sobre el fenómeno (variables sociodemográficas, clínicas, obstétricas, etc.), así como por realizar mediciones en un solo momento temporal. Por otra parte, McGrath, Lingley-Pottie, Emberly, Thurston y McLean (2009) utilizaron un formato de intervención presencial con seguimiento y screening basado en telellamada, implementado por madres veteranas que ejercían de modelos de las futuras mamás para aprender sobre el cuidado de los bebés y el autocuidado durante este periodo vital eminentemente conectado a la mujer. A pesar de que los resultados del estudio aun no se han publicado, el objetivo de este proyecto es cambiar y reducir el diagnóstico de la DPP basándose en la técnica del modelado.

Al margen de estos estudios, no hemos encontrado más estudios (regionales, nacionales o internacionales) que, en una muestra de mujeres embarazadas, se haya realizado una evaluación psicológica autoaplicada online con el objetivo de prevenir la aparición de psicopatología emocional.

Teniendo en cuenta la necesidad de homogeneizar los factores psicosociales que conllevan al desarrollo de trastornos del estado de ánimo en la mujer gestante, su alta prevalencia durante este periodo, y las ventajas que conllevan el uso de los programas e-Health, disponemos de la suficiente base teórica para justificar el proyecto que hemos diseñado. Dicho proyecto, plantea el diseño y desarrollo de una aplicación online, llamada MMF, que sea accesible y de fácil uso y que puede ser un medio de evaluación, diagnóstico y seguimiento eficaz para garantizar la prevención de la DPP antes y después del parto.

\section{Objetivos aplicados de nuestro proyecto: MamáFeliz}

1. Desarrollar una aplicación on line que, de manera autoaplicada, pueda:

- Evaluar medidas sociodemográficas, médicas, psicosociales y psicopatológicas.

- Identificar mujeres embarazadas que cumplan alguno de los factores de riesgo descritos en la literatura científica.

- Identificar mujeres que cumplan criterios diagnósticos (DSM-IV) de episodio depresivo, antes o después del parto.

2. Validar la aplicación MMF como herramienta eficaz para la evaluación, diagnóstico y derivación alcanzando criterios óptimos de sensibilidad y especificidad.

3. Realizar un estudio longitudinal sobre el estado emocional de la mujer durante el embarazo y el posparto.

4. Identificar las variables que actúan como predictoras de la DPREN, TPP y DPP.

5. Proponer, en base a los resultados obtenidos en el estudio, un programa de intervención psicológico cognitivo-comportamental online para la prevención de las alteraciones del estado de ánimo en las mujeres embarazadas y aquellas que han dado a luz.

\section{Metodología}

La muestra mínima total que establecemos es de 924 mujeres para que los resultados obtenidos cuenten con suficiente rigor estadístico. Se seleccionarán aleatoriamente 200 mujeres, 100 con diagnóstico de depresión según MMF y 100 sin diagnóstico, para ser reevalua- 
das por un clínico. Denominaremos grupo control al compuesto por mujeres embarazadas que no cumplen criterios de ningún factor de riesgo ni desarrollen un episodio depresivo antes o después del parto, grupo subclínico a aquellas embarazadas que cumplen criterios de uno o más factores de riesgo para el desarrollo de alteraciones del estado de ánimo pero que no las han desarrollado durante o después del parto y grupo clínico, al grupo formado por mujeres que desarrollen alteraciones del estado de ánimo durante el embarazo o tras el parto.

Se utilizará estadística paramétrica mediante el paquete estadístico para las ciencias sociales en su versión 19.0 (SPSS 19.0, SPSS Inc, Chicago, IL). Se realizarán análisis de frecuencias y descriptivos para describir cada una de las muestras. Para la comparación de medias entre los distintos momentos de evaluación (Pre, Seguimiento 1, 2 y 3) y entre los grupos: control, subclínico y clínico se utilizará análisis de varianza (ANOVA), se utilizará la prueba post hoc de Tukey y se considerará una $p<0.05$ para determinar la significación estadística. Para estudiar la relación entre factores de riesgo específicos con puntuaciones elevadas en EPDS/BDI-II tras el parto, utilizaremos correlaciones lineales y para la relación entre factores de riesgo específicos con el diagnóstico SCID-I, regresión logística. Para la identificación de predictores de alteraciones en el estado de ánimo después del parto, se utilizará el análisis de regresión lineal stepwise. Para evaluar la validez de la aplicación MamáFeliz se calculará la sensibilidad y especificidad con el índice kappa de Cohen. Se computarán los tamaños del efecto aplicando el coeficiente de determinación para las regresiones, «eta» parcial para ANOVAS y «d» de Cohen para comparaciones de medias.

\section{Procedimiento}

Tras la revisión de la literatura científica en el campo de la depresión prenatal y perinatal y el estudio de los programas e-Health que han demostrado eficacia, nos pusimos en contacto con una empresa de ingeniería informática para desarrollar la aplicación MMF a partir de nuestras ideas y necesidades.

Una vez diseñada, desarrollada y testada la aplicación informática, empezamos a contactar con diferentes centros de salud y hospitales de las diferentes comunidades autónomas que quisieran participar en el estudio. Tras la obtención del dictamen favorable por parte de los comités éticos de investigación clínica y la firma de colaboración de los organismos participantes, se les hace entrega, a los profesionales sanitarios implicados (matronas, ginecólogos, etc.), de los códigos de participación para las mujeres embarazadas que deseen formar parte del estudio. En el documento que se les entrega, aparece una breve explicación del proyecto, la dirección web donde deben entrar (www.mamafeliz.es) y el código para registrarse en la aplicación. Una vez registradas, como podemos ver en las imágenes 1 y 2 que mostramos a continuación, deberán leer y aceptar los documentos de protección de datos y confidencialidad, para así poder iniciar su evaluación en la aplicación informática. 


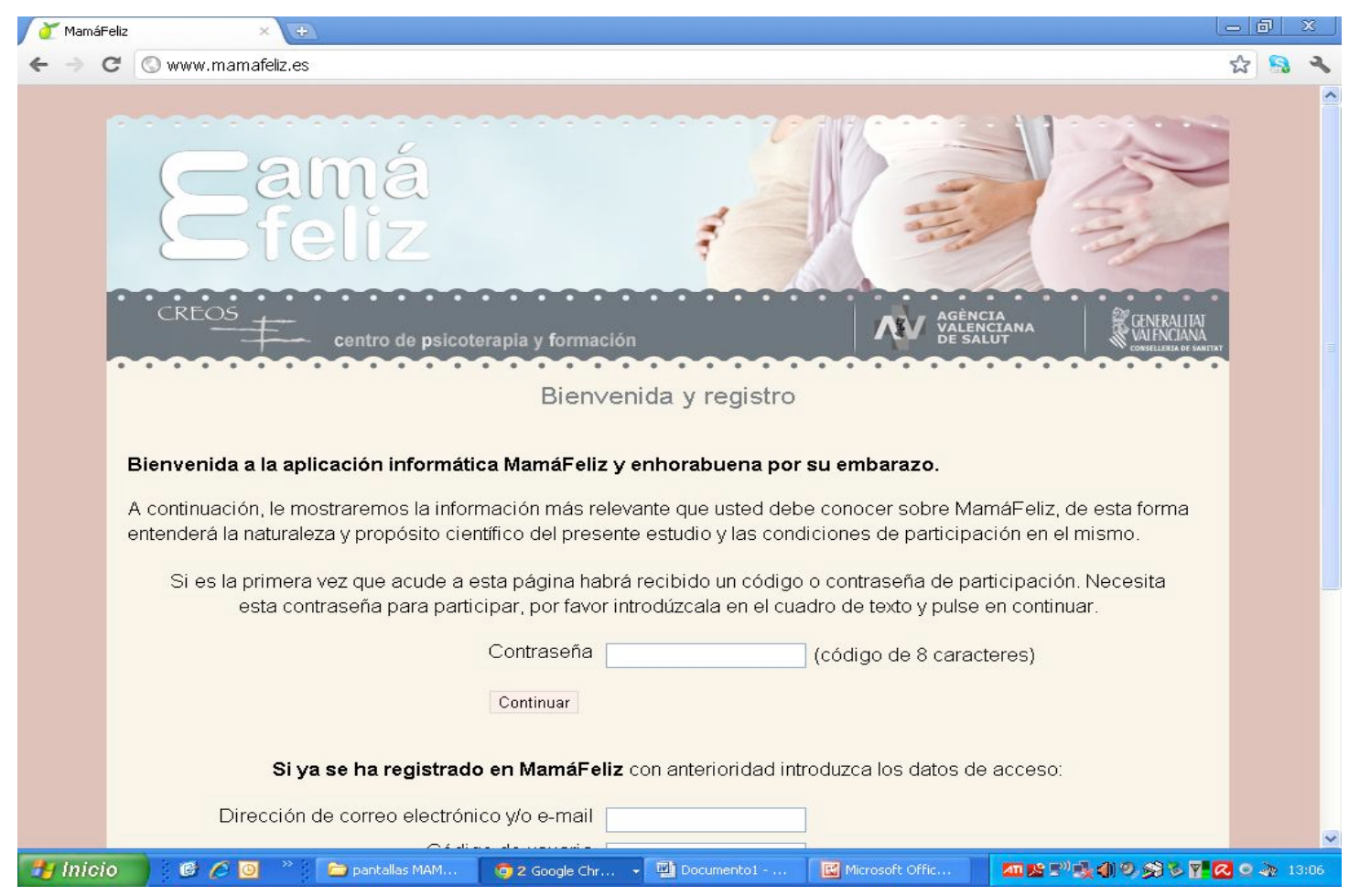

Imagen 1. Bienvenida a la aplicación

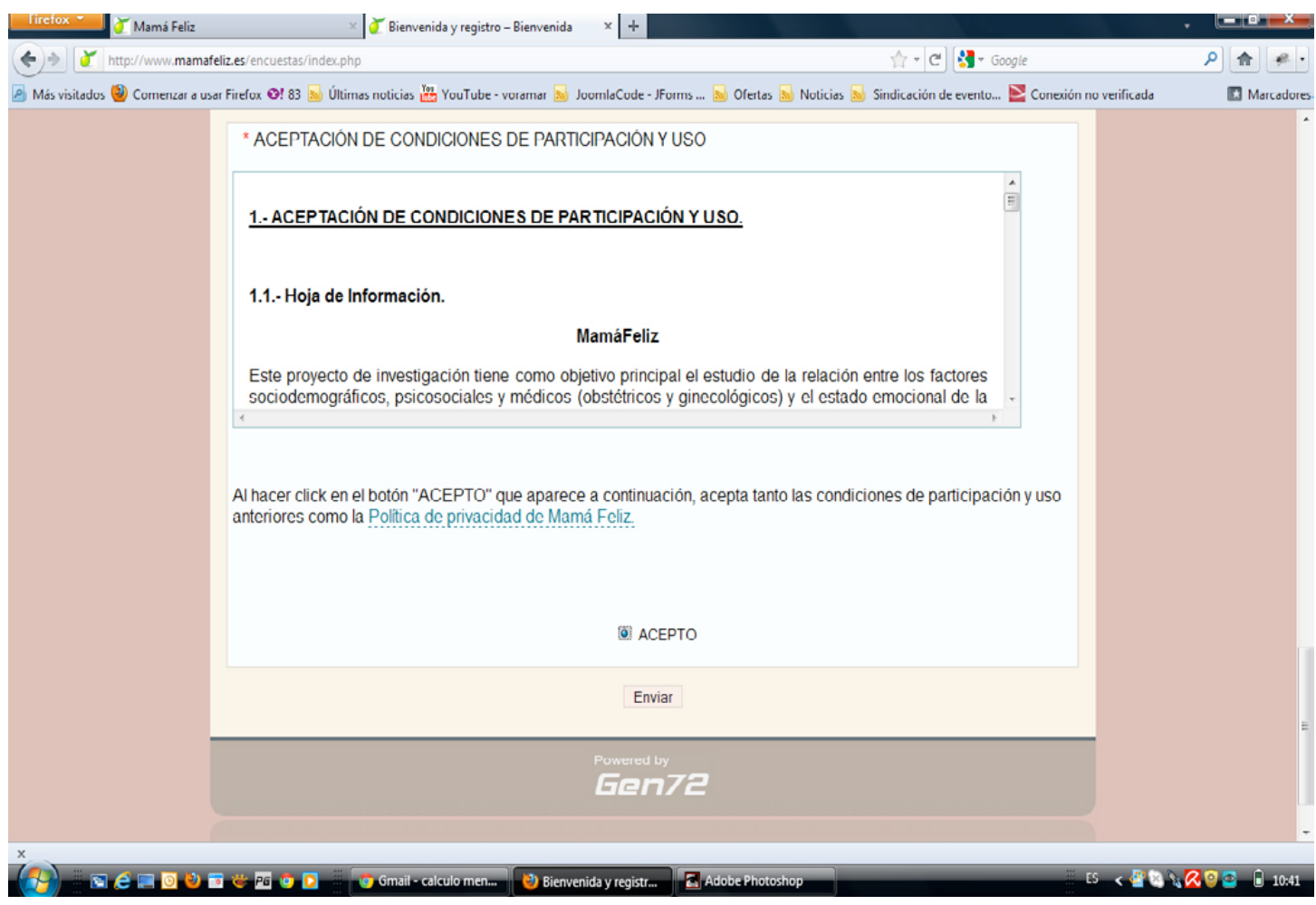

Imagen 2. Aceptación de condiciones de participación (Hoja de Información, Consentimiento Informado y Protección de Datos) 
En este momento es, como observamos en la imagen 3, cuando las usuarias deberán empezar a cumplimentar sus datos personales, sociodemográficos y obstétricos, una entrevista clínica y las distintas medidas psicológicas que rastrean los factores de riesgo identificados en la literatura. La aplicación consta de cinco periodos de evaluación, dos antes del parto (1. ${ }^{\circ}$ entre la semana $16-24$ y $2 .^{\circ}$ entre la 30-36) y tres después del parto (a las 2, 4 y 12 semanas después del parto).

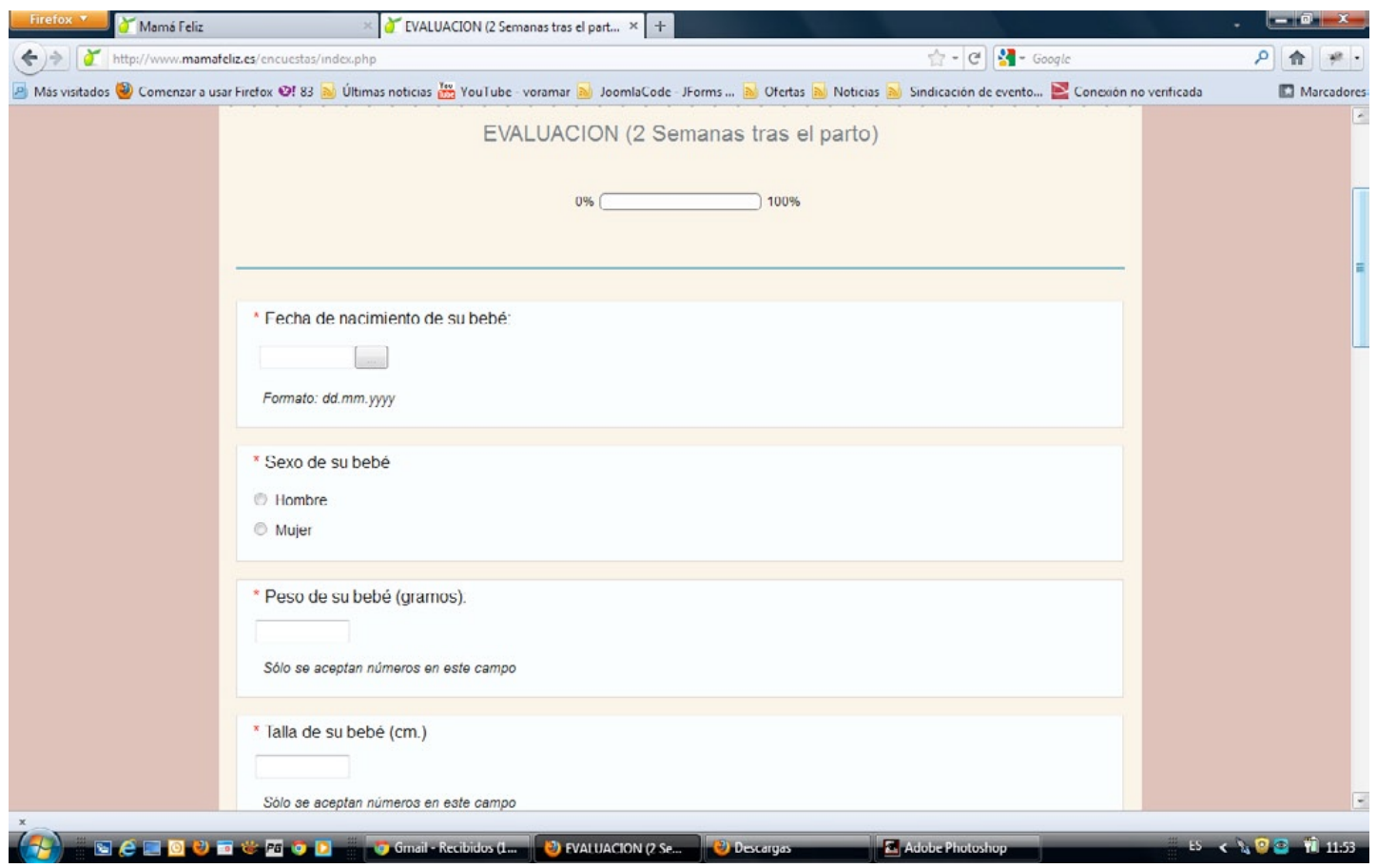

Imagen 3. Cumplimentación datos sociodemográficos y evaluación obstétrica, clínica y psicológica en MMF

Un factor innovador y esencial en la aplicación es, que teniendo en cuenta la semana de gestación en la que la usuaria se encuentra, la aplicación calcula y notifica a cada participante (según su preferencia vía email o sms) el momento en que debe acceder a la aplicación para volver a ser evaluada según sus datos obstétricos. De este modo, podemos analizar la evolución de diferentes medidas (especialmente de los factores de riesgo) a lo largo de todo el embarazo y hasta 3 meses después del parto.

Las participantes que presenten sintomatología depresiva serán informadas y derivadas a su especialista médico y, aquellas que no tengan ninguna alteración emocional, serán informadas de su estado emocional óptimo.

\section{Conclusiones}

La OMS (1995) manifiesta que es esencial disponer de protocolos basados en evidencia para asegurar buenas prácticas y, en este sentido, también desde la Estrategia Nacional de Salud Sexual y Reproductiva (ENSSR) se insta a la investigación, innovación y buenas prácticas con el objetivo de «mejorar la calidad y calidez de la atención en el embarazo, parto, na- 
cimiento, puerperio y periodo neonatal incorporando prácticas innovadoras de demostrada eficacia».

A modo de resumen podemos decir que existe suficiente evidencia científica para plantear, por una parte, que la investigación científica ha identificado los factores de riesgo psicosociales que influyen en el desarrollo de alteraciones emocionales antes y después del parto (DPREN, TPP y DPP, fundamentalmente) y por otra parte, que las aplicaciones web o vía internet son eficaces para la evaluación, la prevención y el tratamiento de trastornos psicológicos.

Nuestro proyecto de investigación, señala la necesidad de realizar una evaluación exhaustiva a nivel psicosocial, obstétrico y psicopatológico de una muestra de mujeres embarazadas con el fin de identificar aquellas mujeres que presentan uno o más factores de riesgo para el desarrollo de psicopatología emocional (DPREN, TPP y DPP, fundamentalmente) antes o después del parto. En el caso de que aparezca sintomatología depresiva previamente (DPREN) o tras el parto (PTT O DPP) es necesaria la intervención rápida para aliviar, lo antes posible, el malestar psicológico en la madre y las repercusiones en su bebé, la pareja y la familia.

Hipotetizamos que una aplicación web accesible y de fácil uso podría ser un medio de evaluación eficaz para la prevención de las alteraciones emocionales antes y después del parto. Además, siguiendo los estándares de este tipo de intervenciones psicológicas, se tomarían en consideración los tres aspectos que la literatura especializada reconoce como garantía de calidad: la relevancia y la eficacia de la intervención para el grupo de pacientes objetivo, los aspectos éticos y garantías, y el diseño y navegabilidad de la aplicación (Whitehead y Proudfoot, 2010). Este proyecto permite el abordaje preventivo de las alteraciones emocionales del periodo del post-parto en todos los niveles de prevención de los trastornos mentales (primaria, secundaria y terciaria), ya que el seguimiento de las variables psicológicas y psicopatológicas en tres momentos a lo largo de las 12 semanas posteriores al momento del parto así lo permitiría.

\section{Referencias bibliográficas}

Andersson, G., Bergstrom, J., Hollandare, F., Carlbring, P., Kaldo, V. \& Ekselius, L. (2005). Internet-based self-help for depression: randomized controlled trial. British Journal of Psychiatry, 187, 456-462.

Andersson, G., Carlbring, P., Holmstrom, A., Sparthan, E., Furmark, T., Nilsson-Ihrfelt, E. et al. (2006). Internetbased self-help with therapist feedback and in vivo group exposure for social phobia: A randomized controlled trial. Journal of Consulting and Clinical Psychology, 74, 677-686.

Andersson, G. (2009). Using the internet to provide cognitive behavior therapy. Behavior Research Therapy, 47, 389-391.

Bashiri, N. \& Spielvogel, A. M. (1999). Postpartum Depression: a cross-cultural perspective. Psychiatry Update, 3 (6), 82-87.

Beck, C. T. (2001). Predictors of postpartum depression: an update. Nurs Res, 50, 275285.

Bennett, N. L., Casebeer, L. L., Zheng, S. \& Kristofco, R. (2006). Information-seeking behaviors and reflective practice. J Contin Educ Health Prof.; 26(2): 120-127

Bennett, N. L., Casebeer, L. L., Kristefeo, R. E. \& Strasser, S. M. (2004). Physicians' Internet informationseeking behaviours. J Contin Educ Health Prof.; 24: 31-38.

Choque Larrauri, R. (2010). Nuevas competencias tecnológicas en Información y Comunicación. Perú: CONCYTEC. 
Cohen, L. S., Wang, B., Nonacs, R., Viguera, A. C., Lemon, E. L. \& Freeman, M. P. (2010). Treatment of mood disorders during pregnancy and postpartum; Psychiatric Clinics of North America, 2, (33), 273-293.

Cox, J. L., Holden, J. M. \& Sagovsky, R. (1987). Detection of postnatal depression: Development of the 10-item Edinburgh Postnatal Depression Scale. British Journal of Psychiatry, 150, 782-786.

Dosset, E. C. (2008). Perinatal depression. Obstet Gynecol Clin North Am, 35, 419-434.

Edwards, B., Galletly, C., Semmier-Booth, T. \& Dekker, G.(2008). Does antenatal screening for psychological risk factors predict postnatal depression? A follow-up study of 154 women in Adelaide, South Africa. The Australian and New Zealand journal of Psychiatry, 42, 51-55.

ENSSR (2010). Estrategia Nacional en Salud Sexual y Reproductiva. Ministerio de Sanidad y Política Social. Madrid: Gobierno de España.

EU (2005) Green Paper. Improving the mental health of population: Towards a strategy on mental health for the European Union. Brussels: European Communities, Health and Consumer Protection Directorate-General.

Field, T. (2011). Prenatal depression effects on early development: A review. Infant Behavior and Development, 34, 1-14.

García-Esteve, L., Ascaso, C., García, M. \& Tamayo, O. (2003). Maternidad y Trabajo: impacto de la morbilidad psiquiátrica puerperal en la adaptación al año de las madres trabajadoras. Trabajo de Investigación. Universidad de Barcelona.

Gaynes, B. N., Gavin, N., Meltzer-Brody, S. et al. (2005). Perinatal depression: prevalence, screening accuracy and screening outcomes. Evidence Report: Technology Assessment 119, 1-8. Rockvill, MD: Agency for Health Research and Quality.

Glavin, K., Smith, L., Sorum, R. \& Ellefsen, B. (2010). Redesigned community postpartum care to prevent and treat postpartum depression in women - a one year follow-up study. Journal of Clinical Nursing, 19, 3051-3062.

Hampton, T. (2006). Researchers provide psychiatric care from afar. Journal of the American Medical Association, 295, 21-22.

Hearn, G., Iliff, A., Jones, I., Kirby, A., Ormiston, P., Parr, P., Rout, J. \& Wardman, L. (1998). Postnatal depression in the community. The British Journal of General Practice, 48, 1064-1066.

Hosman, C., Jané-Llopis, E. (1999). The evidence of health promotion effectiveness: shaping public health in Europe. In: Political Changes 2 Mental Health (pp. 29-41). Brussels: ECSC-EC-EAEC.

Huizink, A. C., Mulder, J. H. \& Buitelaar, J. H. (2004). Prenatal stress and risk for psychopathology: specific effects or induction of general susceptibility. Psychological Bulletin, 130, 115-142.

Jané-Llopis, E. (2004). La eficacia de la promoción de la salud mental y la prevención de los trastornos mentales. Revista de la Asociación Española de Neuropsiquiatria, 89, 67-78.

Kelly, R. H., Zatzick, D. F. \& Anders, T. F. (2001). The detection and treatment of psychiatric disorders and substance use among pregnant women cared for in obstetrics. American Journal of Psychiatry, 158,213-219.

Le, H., Perry, D. F. \& Sheng, X. (2009). Using the Internet to Screen for Postpartum Depression. Maternal Child Health Journal, 13, 213-221.

McGrath, P. J., Lingley-Pottie, P., Emberly, D. J., Thurston, C. \& McLean, C. (2009). Integrated knowledge translation in mental health: family help as an example. J Can Acad Child Adolesc Psychiatry, 18(1):30-37. 
Milgrom, J., Gemmill, A. W., Bilszta , J. L., Hayes, B., Barnett, B., Brooks, J. et al. (2008). Antenatal risk factors for postnatal depression:A large prospective study. Journal of Affective Disorders, 108, 147-157.

Mitchell, J., Stanimirovic, R., Klein, B. \& Vella-Brodrick, D. (2009). A randomised controlled trial of self-guided internet interventions promoting well-being. Computers in Human Behavior, 25, 749-760

Mrazek, P. J. \& Hall, M. (1997). A policy perspective on prevention. American Journal of community psychology, 25, 221-226.

Navarro, P. \& García-Esteve, LI., Ascaso, C., Aguado, J. y cols. (2008). Non-psychotic psychiatric disorders after childbirth: Prevalence and comorbidity in a community sample. Journal of affective disorders; 109, (1); 171-176.

Oates, M. R., Cox, J. L., Neema, S., Asten, P., Glangeaud-Freudenthal, N., Figueiredo, B. et al. (2004). Postnatal depression across countries and cultures: a qualitative study. British Journal of Psychiatry, 46, 10-16.

OMS (1986). Carta de Ottawa para la Promoción de la Salud. Génova: Organización Mundial de la Salud.

OMS (2008) Pacto Europeo para la salud mental y el bienestar. Conferencia de alto nivel de la UE: Juntos por la salud mental y el bienestar. Bruselas, 2008.

Pier, C., Austin, D., Klein, B., Mitchell, J., Schattner, P., Ciechomski, I. et al. (2008). Evaluation of internet-based behavioural therapy for panic disorder in general medical practice. Mental Health in Family Medicine, 5, 29-39.

Pop, V. J. M, Essed, G. G. M, De Geus, C. A., Van Son, M. M. \& Komproe, I. H. (1993). Prevalence of postpartum depression or is it postpuesperium depression?, Acta Obstet. Gynecol. Scand, 72, 354-358.

Proudfoot, J., Goldberg, D., Mann, A., Everitt, B., Marks, I. \& Gray, J. (2003). Computerized, interactive, multimedia cognitive-behavioural program for anxiety and depression in general practice. Psychological Medicine, 33, 217-227.

Ritterband, L., Cox, D., Walker, L., Kovatchev, B., McKnight, L., Patel, K. et al. (2003). An Internet intervention as adjunctive therapy for pediatric encopresis. Journal of Consulting and Clinical Psychology, 71, 910-917.

Soto-Pérez, F., Franco, M., Monardes, C. \& Jiménez, F. (2010). Internet y psicología clínica: revisión de las ciber-terapias. Revista de Psicopatologia y Psicologia clínica, 15(1), 19-37.

Spittaels H. \& de Bourdeaudhuij, I. (2006). Implementation of an online tailored physical activity intervention for adults in Belgium. Health promotion International, 221, 311-319.

Tortajada, S., García-Gómez, J. M., Vicente, J., Sanjuan, J., De Frutos, R., Martín-Santos, R., García-Esteve, L., Gornemann, I., Gutiérrez-Zotes, A., Canellas, F., Carracedo, A., Gratacós, M., Guillamat, R., Baca-García, E. \& Robles, M. (2009). Prediction of postpartum depression using multilayer perceptrons and pruning. Methods of information in medicine, 48 (3): 291-298.

U. S. DHHS (2000). Healthy people 2010: Understanding and improving health. United States Department Health and Human Services Washington DC: U. S. Government Printing Office.

Walters, S. T., Miller, J. E. \& Chiauzzi, E. (2005). Wired for wellness: e-interventons for addressing college drinking. Journal of Substance Abuse Treatment, 29, 139-145.

Walters, S. T., Wright, J. A. \& Shegog, R. (2006). A review of computer and internet-based interventions for smoking behaviour. Addictive Behaviors, 31, 264-277.

Weissman, M. M. \& Olfson, M. (1995). Depression in women: implications for health care research. Science, 269, 799-801. 
Whitehead, L. \& Proudfoot, J.(2010). Standards and operating guidelines for internet interventions. In: J. Bennet-Levy et. al. (eds.) Oxford guide to low intensity CBT interventions. 247-252. New York: Oxford University Press. 